\title{
One-stage nipple and breast reconstruction using a deep inferior epigastric perforator flap after a skin- sparing mastectomy
}

\author{
Hyun Jun Cho, Hyo Jeong Kwon, Suk-Ho Moon, Young Joon Jun, Jong Won Rhie, \\ Deuk Young Oh \\ Department of Plastic and Reconstructive Surgery, College of Medicine, The Catholic University of Korea, Seoul, Korea
}

Background Nipple reconstruction is usually performed as a delayed procedure in patients with breast cancer who undergo skin-sparing mastectomy and breast reconstruction surgery using a deep inferior epigastric perforator (DIEP) flap. The authors designed this study to evaluate the utility of breast reconstruction based on a DIEP flap and immediate nipple reconstruction.

Methods A retrospective review was conducted of all patients who underwent breast reconstruction performed by a single plastic surgeon from October 2016 to June 2018. Through a questionnaire and chart review, we compared surgical results and complications in cases of single-stage nipple reconstruction after skin-sparing mastectomy $(n=17)$ with patients who underwent delayed nipple reconstruction after skin-sparing mastectomy, modified radical mastectomy, or simple mastectomy $(n=7)$.

Results In a subjective analysis using clinical photos, the immediate nipple reconstruction group had higher scores than their counterparts in an evaluation of the nipple-areolar complex (NAC) (NAC placement, 3.34 vs. 3.04; nipple projection, 3.05 vs. 3.03; nipple size, 3.30 vs. 3.29). No significant differences between the groups were found in terms of complications.

Conclusions Simultaneous nipple reconstruction is a reliable surgical method with economic advantages. No differences were found in terms of outcomes and complications in comparison to delayed reconstruction. Therefore, surgeons can consider simultaneous nipple reconstruction without particular concerns about asymmetry or necrosis.

Keywords Mammaplasty / Mastectomy / Nipples
Correspondence: Deuk Young Oh Department of Plastic and Reconstructive Surgery, Seoul St. Mary's Hospital, College of Medicine, The Catholic University of Korea, 222 Banpo-daero, Seocho-gu, Seoul 06591, Korea

Tel: $+82-2-2258-2842$

Fax: +82-2-594-7230

E-mail: ohdeuk1234@hanmail.net

Received: May 7, $2019 \bullet$ Revised: October 24, $2019 \bullet$ Accepted: October 24, 2019

pISSN: 2234-6163 • elSSN: 2234-6171 • https://doi.org/10.5999/aps.2019.00598 • Arch Plast Surg 2020;47:26-32

This article was presented at PRS Korea 2018 on November 9-11, 2018, in Seoul, Korea.

\section{INTRODUCTION}

In the past, mastectomy was the sole procedure performed to treat breast cancer. However, in recent years, treatment strategies for breast cancer have evolved in a way that balances onco- logic safety and aesthetic considerations; as a result of these developments, breast reconstruction is widely used in mastectomy patients. Nipple-sparing mastectomy (NSM) and skin-sparing mastectomy (SSM) constitute major advances in breast cancer surgery [1]. These procedures, often called conservative mas- 
tectomies, reflect a paradigm shift in breast cancer treatment. In SSM, the nipple-areolar complex (NAC) is removed, so a process for making nipples is necessary [2].

Nipple reconstruction has usually been performed as a secondary procedure, about 6 months after the primary breast reconstruction, to allow flap stabilization and breast symmetry [3]. However, a disadvantage of performing separate procedures is that dysmorphic changes can occur in the reconstructed breast as a result of nipple reconstruction using a flap, and it may not be possible to create a sufficiently large nipple.

In SSM, the position of the nipple is expected to remain almost unchanged because the location of the areola is predefined. In addition, in breast reconstruction procedures using a deep inferior epigastric artery (DIEP) flap, the abundance of available skin means that a sufficiently large nipple can be created. For these reason, we attempted to perform reconstruction of the nipple and breast in a single stage. The purpose of this study was to review our experience of one-stage nipple and breast reconstruction using a DIEP flap following SSM.

\section{METHODS}

A retrospective review was conducted of all patients who under- went breast reconstructions performed by a single surgeon (DYO) from October 2016 to June 2018. The mastectomy and axillary clearance were performed by general surgeons. In total, 165 patients underwent breast reconstruction with a DIEP flap after mastectomy, among whom 163 were included in the analysis, with the exception of two patients who underwent bilateral procedures. Immediate breast reconstruction was performed in 135 of these patients, among whom 45 underwent SSM (Fig. 1). Of these, 35 patients underwent immediate nipple reconstruction with a modified $\mathrm{C}-\mathrm{V}$ flap. Of the 35 patients who underwent immediate nipple reconstruction, follow-up with clinical pictures was possible in 17 patients. Only two of the other 10 patients underwent delayed nipple reconstruction, while the remaining eight did not undergo nipple reconstruction at all. Because these numbers were too small, we included patients who underwent delayed nipple reconstruction after simple mastectomy or modified radical mastectomy (MRM). Thus, we performed a subjective analysis of 17 patients who underwent onestage nipple reconstruction and 12 patients who underwent delayed nipple reconstruction after simple mastectomy, MRM, or SSM. Of the 12 patients who underwent delayed reconstruction, follow-up with clinical pictures was possible in seven. Therefore, we compared 17 patients in the experimental group

\section{Fig. 1. Flowchart of patients by mastectomy type}

DIEP, deep inferior epigastric perforator; MRM, modified radical mastectomy.

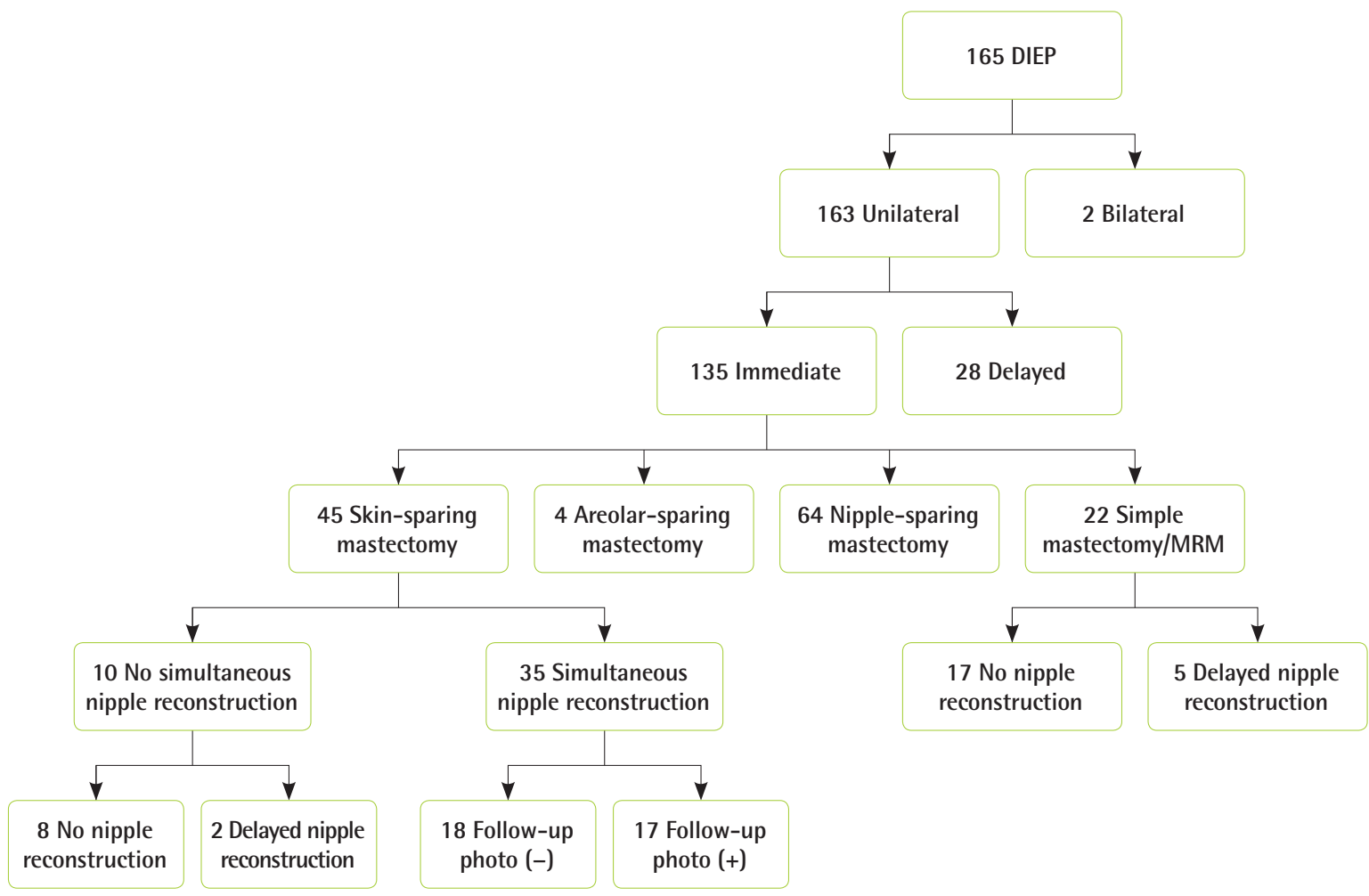


Table 1. Demographics of enrolled patients

\begin{tabular}{|c|c|c|c|c|c|c|}
\hline No. & Age (yr) & Breast shape & Mastectomy type & Nipple reconstruction & Follow-up (mon) & Nipple complications \\
\hline 1 & 51 & Normal & SSM & Immediate & 4 & None \\
\hline 2 & 55 & Ptotic & SSM & Immediate & 15 & None \\
\hline 3 & 43 & Normal & SSM & Immediate & 25 & None \\
\hline 4 & 63 & Ptotic & SSM & Immediate & 9 & None \\
\hline 5 & 44 & Normal & SSM & Immediate & 22 & Partial necrosis \\
\hline 6 & 45 & Ptotic & SSM & Immediate & 9 & None \\
\hline 7 & 57 & Ptotic & SSM & Immediate & 5 & None \\
\hline 8 & 48 & Ptotic & SSM & Immediate & 15 & None \\
\hline 9 & 48 & Ptotic & SSM & Immediate & 12 & None \\
\hline 10 & 27 & Normal & SSM & Immediate & 14 & None \\
\hline 11 & 55 & Ptotic & SSM & Immediate & 17 & None \\
\hline 12 & 38 & Large & SSM & Immediate & 17 & None \\
\hline 13 & 41 & Thin & SSM & Immediate & 14 & None \\
\hline 14 & 48 & Ptotic & SSM & Immediate & 22 & None \\
\hline 15 & 42 & Normal & SSM & Immediate & 5 & None \\
\hline 16 & 41 & Normal & SSM & Immediate & 12 & None \\
\hline 17 & 42 & Ptotic & SSM & Immediate & 14 & None \\
\hline 18 & 38 & Small & Simple mastectomy & Delayed & 19 & None \\
\hline 19 & 57 & Ptotic & MRM & Delayed & 28 & Shrinkage \\
\hline 20 & 40 & Normal & Simple mastectomy & Delayed & 26 & Disfiguration \\
\hline 21 & 41 & Normal & Simple mastectomy & Delayed & 28 & Shrinkage, disfiguration \\
\hline 22 & 36 & Normal & MRM & Delayed & 28 & None \\
\hline 23 & 42 & Normal & MRM & Delayed & 10 & None \\
\hline 24 & 36 & Small & Simple mastectomy & Delayed & 23 & None \\
\hline
\end{tabular}

with seven in the control group (Table 1).

To analyze the results, we conducted surveys. To evaluate complications, three items (nipple necrosis, shrinkage, and disfigurement) were assessed through chart review. The follow-up period ranged from 4 months to 28 months (mean, 16.4 months).

\section{Surgical technique}

During SSM performed by a general surgeon, we harvested a DIEP flap considering the overall breast shape and volume. In most cases, including patients with ptotic breasts, we inset the flap in the vertical direction and used the thoracodorsal system as recipient vessels. However, if the breast was too wide, or if the vessel length was insufficient, we inset the flap in the horizontal direction.

After setting the DIEP flap in the mastectomy site, we fixed it to the remaining upper and medial fascia of the chest wall with 3-0 Vicryl sutures. Next, the patient was placed in the sitting position to evaluate symmetry. Considering the position and size of the opposite breast, the NAC defect was marked on the skin paddle of the DIEP flap. We designed a modified C-V flap on the skin paddle using the available skin. Loss of projection of the new nipple should always be anticipated, so we overcorrected by $30 \%$ of the desired result $[4,5]$. At this time, we had a suffi- cient skin envelope, so we could make a larger nipple than that on the contralateral side in preparation for nipple atrophy. Considering the location of the perforator, we designed a $\mathrm{C}$ flap on the opposite side of the perforator (Fig. 2). Next, we designed the areola and performed de-epithelization of the remnant skin paddle (Fig. 3).

\section{Questionnaire survey}

The reconstructive and cosmetic results were evaluated by two specialists in plastic surgery, two residents in plastic surgery, and one medical student. The assessment was based on six items (NAC placement, nipple projection, nipple size, breast mound projection, breast mound symmetry, and overall breast reconstruction) with a scale of 1 to 4 points (1, poor; 2 , fair; 3 , good; 4 , excellent). Of the six items, three were related to the nipple. All evaluators were given the same standardized photographs and were asked to complete the items.

\section{Statistical analysis}

Data are presented as mean \pm standard deviation. The Wilcoxon rank-sum test and the Fisher exact test were used where appropriate to evaluate the statistical significance of differences between the two groups; P-values $<0.05$ were considered to indi- 


\section{Fig. 2. The nipple design: modified C-V flap}

Considering the perforator location, we designed a "C" flap on the opposite direction of the perforator.

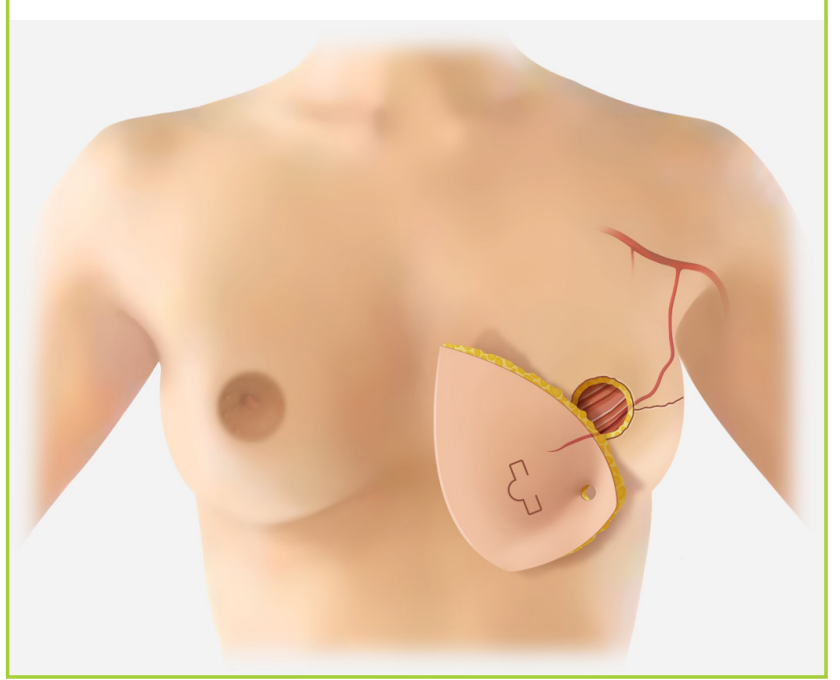

cate significant differences. SAS version 9.4 (SAS Institute Inc., Cary, NC, USA) was used for database management and for the statistical analysis.

\section{RESULTS}

For the subjective analysis, we conducted a questionnaire survey containing six items based on clinical photographs of the 17 patients who underwent immediate nipple reconstruction and the seven patients who underwent delayed reconstruction. The patients who underwent immediate nipple reconstruction had good results on the nipple-related questions. For the symmetry of NAC placement, the immediate reconstruction group received a score of 3.34 points and the delayed reconstruction group received a score of 3.04 points. The score for maintenance of nipple projection was 3.05 points in the immediate reconstruction group and 3.03 points in the delayed reconstruction group. The score for symmetry of nipple size was 3.30 points in the immediate reconstruction group and 3.29 points in the delayed reconstruction group. However, none of these differences were statistically significant (Table 2).

No major complications, such as total flap necrosis, occurred in either group. Partial nipple necrosis occurred in one of the 17 patients in the immediate reconstruction group, and in none of the patients in the delayed reconstruction group. Nipple shrinkage and disfiguration occurred in two of the seven patients in the delayed reconstruction group and in none of the patients in the immediate reconstruction group. In short, the rate of nipplerelated complications was low in the immediate reconstruction group, but there were no statistically significant between-group

\section{Fig. 3. New nipple-areolar complex}

After making the nipple, we designed the areola and performed deepithelization.

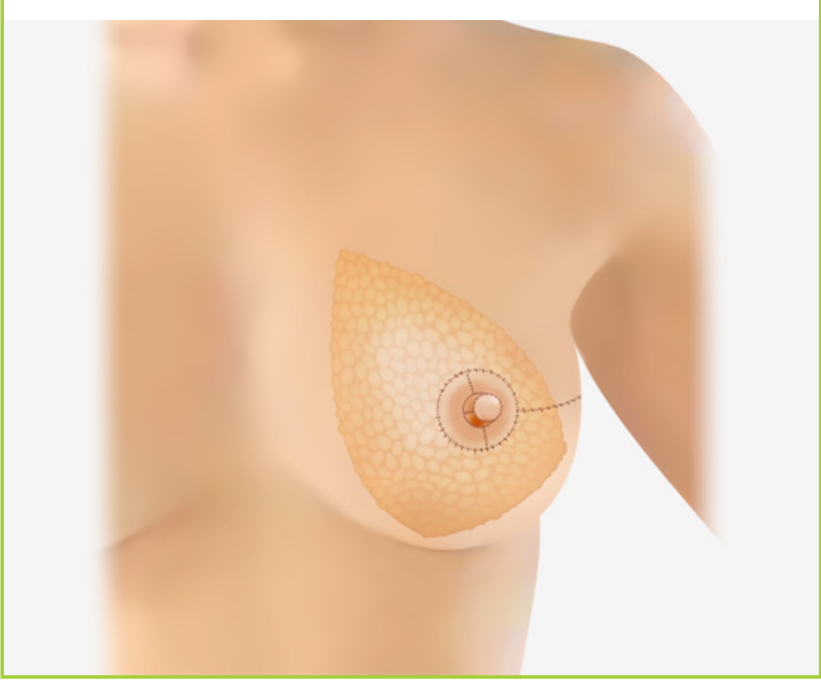

Table 2. Photograph-based subjective assessments

\begin{tabular}{|c|c|c|c|}
\hline & $\begin{array}{l}\text { Immediate } \\
(\mathrm{n}=17)\end{array}$ & $\begin{array}{c}\text { Delayed } \\
(n=7)\end{array}$ & P-value ${ }^{a)}$ \\
\hline NAC placement & $3.34 \pm 0.88$ & $3.04 \pm 1.07$ & 0.386 \\
\hline Nipple projection & $3.05 \pm 0.77$ & $3.03 \pm 1.14$ & 0.867 \\
\hline Nipple size & $3.30 \pm 0.79$ & $3.29 \pm 0.81$ & 1 \\
\hline Breast mound projection & $3.27=$ & $3.32 \pm$ & 0.762 \\
\hline Breast mound symmetry & $3.02 \pm$ & $3.04 \pm$ & 1 \\
\hline Overall breast reconstruction & $3.21 \pm 0.69$ & $3.12 \pm 0.90$ & 0.741 \\
\hline \multicolumn{4}{|c|}{$\begin{array}{l}\text { Values are presented as mean } \pm \text { SD. Two specialists in plastic surgery, two } \\
\text { residents in plastic surgery, and one medical student evaluated standardized } \\
\text { photographs on a scale of } 1 \text { to } 4 \text { points (1, poor; } 2 \text {, fair; } 3 \text {, good; } 4 \text {, excellent). } \\
\text { We used the Wilcoxon rank-sum test and nonparametric test. } \\
\text { NAC, nipple-areolar complex. } \\
\text { alP }<0.05 \text { was considered to be significant. }\end{array}$} \\
\hline
\end{tabular}

Table 3. Postoperative complications

\begin{tabular}{|lccc|}
\hline & Immediate (\%) & Delayed (\%) & P-value $^{\text {a) }}$ \\
\hline Partial nipple necrosis & 5.8 & 0 & 1 \\
Nipple shrinkage & 0 & 28.6 & 0.083 \\
Nipple disfiguration & 0 & 28.6 & 0.083 \\
\hline $\begin{array}{l}\text { The Fisher exact test was performed to test for significant differences between } \\
\text { the two groups in terms of nipple-related complications. } \\
\text { a) }<<0.05 \text { was considered to be significant. }\end{array}$ \\
\hline
\end{tabular}

\section{differences}

Postoperative complications included partial nipple necrosis, shrinkage, and disfiguration. Partial necrosis occurred in 5.8\% of the patients in the immediate reconstruction group and in $0 \%$ of the patients in the delayed reconstruction group. The corresponding proportions for nipple shrinkage and nipple disfiguration were $0 \%$ vs. $28.6 \%$ and $0 \%$ vs. $28.6 \%$, respectively. However, 

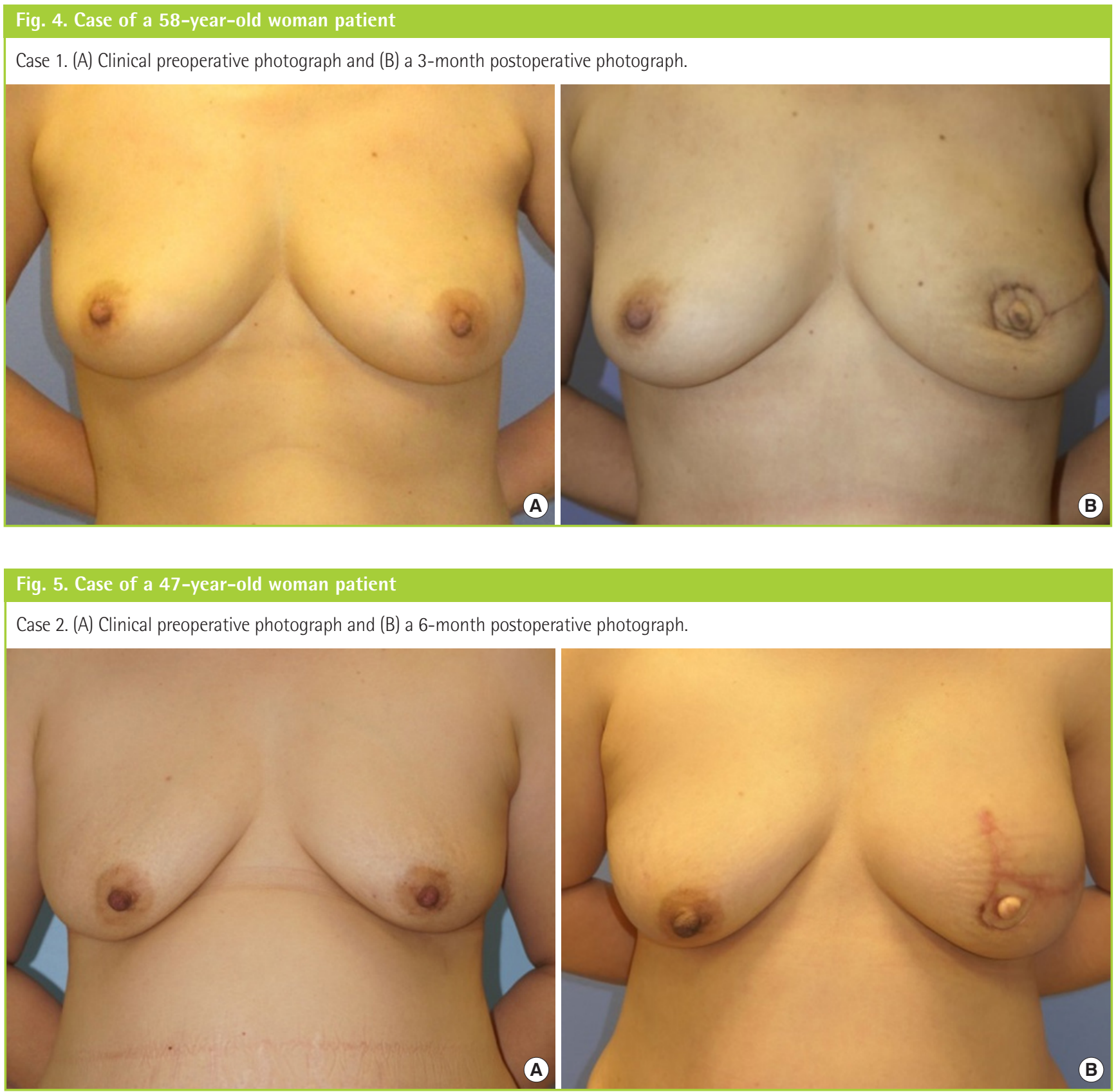

none of these differences were statistically significant (Table 3).

\section{Cases}

\section{Case 1}

A 58-year-old woman underwent SSM and immediate reconstruction with DIEP flap coverage (Fig. 4). A modified C-V flap was used for one-stage nipple reconstruction. At 3 months postoperatively, the shape and position of the reconstructed nipple were similar to those of the contralateral nipple. The height of the nipple was well-maintained, and no complications were observed.

\section{Case 2}

A 47-year-old woman underwent SSM and DIEP flap coverage (Fig. 5). The nipple was reconstructed with a modified C-V flap in one stage. No complications were observed. The height and shape of the reconstructed nipple at 6 months postoperatively were similar to those of the contralateral nipple.

\section{DISCUSSION}

The NAC plays an aesthetically important role in the breast. Therefore, reconstructing the nipple is also an important factor in breast reconstruction procedures. Since Berson first made a 
nipple with a local flap in 1946, various methods have been developed, and there is a growing interest in nipple reconstruction [4]. Bykowski et al. [6] measured satisfaction and quality of life using the BREAST-Q questionnaire among patients who underwent nipple reconstruction. They found that patients' psychosocial well-being and sexual well-being improved after nipple reconstruction, indicating that nipple reconstruction procedures could improve patients' outcomes. Satteson et al. [7] conducted a systematic review of NAC reconstruction and patient satisfaction. Patient satisfaction with nipple reconstruction was high regardless of the surgical technique, and was even higher with NSM.

The timing of nipple reconstruction is important for the final aesthetic outcome [8-10]. However, nipple reconstruction has so far been considered a secondary procedure in breast reconstruction. Traditionally, the nipple is reconstructed at least 6 months after the last revision of reconstructive surgery, because if the reconstruction is performed earlier, the final position of the nipple may be affected by swelling or internal inflammation. Furthermore, it is thought that the flap may be insufficiently stabilized before it nipple reconstruction is carried out [11].

However, a drawback of two-stage reconstruction is that a sufficiently large nipple cannot be made because a small amount of skin is used to create the nipple. Furthermore, in SSM, the portion to be used for the NAC is fixed, and therefore it is expected to be almost unchanged [12]. For these reasons, we performed nipple reconstruction simultaneously with reconstruction with a DIEP flap in patients with SSM and obtained satisfactory results.

The 17 patients who underwent nipple reconstruction in one stage were analyzed using clinical photographs. During the longterm follow-up period, nipple placement, projection, and size were well maintained compared to the contralateral side, but there were no statistically significant differences between the two groups. Major complications, such as DIEP flap loss, did not occur in any cases. In a comparison of nipple-related complications, such as nipple necrosis and shrinkage, there was no significant difference between the two groups.

A well-performed single-stage breast and nipple reconstruction has several advantages. First, it prevents breast disfiguration since the nipple position is set in advance. Second, due to the presence of a sufficient skin remnant, we can make one nipple larger than the other. Therefore, nipple asymmetry due to shrinkage can be prevented. Third, because the breast and nipple are reconstructed in a single operation, fewer procedures are performed, which saves time and may decrease the total financial burden on the patient. Although simultaneous reconstruction incurs additional general anesthesia fees, it has the advantage of saving the additional medical expenses, transportation costs, medical costs, and social costs incurred by a two-stage procedure.

However, there still are some limitations of this study. The major limitation of this study is that we could perform only subjective assessments, because we could not measure objective values such as nipple projection or size. Another limitation is that we compared an SSM group to a group in which SSM, MRM, and simple mastectomy was performed. Although it would have been ideal to compare one-stage and two-stage reconstruction groups in patients who underwent SSM, too few patients would have been enrolled using such a design. Instead, it was necessary to enroll patients who underwent MRM or simple mastectomy. The results would have been statistically more powerful if there were more patients, so a larger study will be needed in the future. Simultaneous nipple reconstruction has the advantages of easy positioning of the nipple and flap direction of the reconstructed nipple, as well as making it possible to create a larger nipple considering shrinkage after reconstruction. It is also an economic and time-saving surgical method. This study revealed that there were no significant differences in complications between the simultaneous nipple reconstruction method and the classical multi-stage nipple reconstruction method.

In conclusion, surgeons can consider simultaneous nipple reconstruction without particular concerns about asymmetry or necrosis.

\section{NOTES}

\section{Conflict of interest}

No potential conflict of interest relevant to this article was reported.

\section{Ethical approval}

The study was approved by the Institutional Review Board of the Catholic University of Korea Seoul St. Mary's Hospital (IRB No. KC19RESI0159) and performed in accordance with the principles of the Declaration of Helsinki. Written informed consents were obtained.

\section{Patient consent}

The patients provided written informed consent for the publication and the use of their images.

\section{Author contribution}

Conceptualization: Cho HJ, Oh DY. Data curation: Kwon HJ, Oh DY. Formal analysis: Moon SH, Oh DY. Methodology: Rhie JW, Oh DY. Project administration: Cho HJ, Kwon HJ. Visualization: Kwon HJ. Writing - original draft: Cho HJ, Kwon HJ. 
Writing - review \& editing: Jun YJ, Oh DY. Approvof final manuscript: all authors.

\section{ORCID}

Hyun Jun Cho https://orcid.org/0000-0003-0353-3606 Hyo Jeong Kwon https://orcid.org/0000-0002-5778-5692 Suk-Ho Moon https://orcid.org/0000-0003-1866-2910 Young Joon Jun https://orcid.org/0000-0001-6537-2460 Jong Won Rhie https://orcid.org/0000-0002-9398-8059 Duek Young Oh https://orcid.org/0000-0003-3499-1554

\section{REFERENCES}

1. Galimberti V, Vicini E, Corso G, et al. Nipple-sparing and skin-sparing mastectomy: review of aims, oncological safety and contraindications. Breast 2017;34 Suppl 1:S82-4.

2. Tremp M, di Summa PG, Schaakxs D, et al. Nipple reconstruction after autologous or expander breast reconstruction: a multimodal and 3-dimensional analysis. Aesthet Surg J 2017;37:179-87.

3. Kristoffersen CM, Seland H, Hansson E. A systematic review of risks and benefits with nipple-areola-reconstruction. J Plast Surg Hand Surg 2017;51:287-95.

4. Sisti A, Grimaldi L, Tassinari J, et al. Nipple-areola complex reconstruction techniques: a literature review. Eur J Surg Oncol 2016;42:441-65.
5. Nimboriboonporn A, Chuthapisith S. Nipple-areola complex reconstruction. Gland Surg 2014;3:35-42.

6. Bykowski MR, Emelife PI, Emelife NN, et al. Nipple-areola complex reconstruction improves psychosocial and sexual well-being in women treated for breast cancer. J Plast Reconstr Aesthet Surg 2017;70:209-14.

7. Satteson ES, Brown BJ, Nahabedian MY. Nipple-areolar complex reconstruction and patient satisfaction: a systematic review and meta-analysis. Gland Surg 2017;6:4-13.

8. Williams EH, Rosenberg LZ, Kolm P, et al. Immediate nipple reconstruction on a free TRAM flap breast reconstruction. Plast Reconstr Surg 2007;120:1115-24.

9. Yoon JS, Chang JW, Ahn HC, et al. Modified C-H flap for simultaneous nipple reconstruction during autologous breast reconstruction: surgical tips for safety and cosmesis. Medicine (Baltimore) 2018;97:e12460.

10. Hyza P, Streit L, Vesely J, et al. New technique of immediate nipple reconstruction during immediate autologous DIEP or MS-TRAM breast reconstruction. Ann Plast Surg 2015; 74:645-51.

11. Nahabedian MY. Nipple reconstruction. Clin Plast Surg 2007;34:131-7.

12. Kim HR, Lim JS, Kim SM, et al. One-stage nipple and breast reconstruction following areola-sparing mastectomy. Arch Plast Surg 2013;40:553-8. 